\title{
BMJ Open Clinical decision-making for spinal manipulation for persistent spinal pain following lumbar surgery: a protocol for a systematic review and meta-analysis of individual participant data
}

\author{
Robert James Trager (D) , ${ }^{1}$ Clinton J Daniels (D) , ${ }^{2}$ Kevin W Meyer (i) , ${ }^{3}$ \\ Amber C Stout, ${ }^{4}$ Jeffery A Dusek (i) ${ }^{1}$
}

To cite: Trager RJ, Daniels CJ, Meyer KW, et al. Clinical decision-making for spinal manipulation for persistent spinal pain following lumbar surgery: a protocol for a systematic review and meta-analysis of individual participant data. BMJ Open 2021;11:e054070. doi:10.1136/ bmjopen-2021-054070

- Prepublication history and additional supplemental material for this paper are available online. To view these files, please visit the journal online (http://dx.doi.org/10.1136/ bmjopen-2021-054070).

Received 02 June 2021 Accepted 29 November 2021

\section{Check for updates}

(C) Author(s) (or their employer(s)) 2021. Re-use permitted under CC BY-NC. No commercial re-use. See rights and permissions. Published by BMJ.

${ }^{1}$ Connor Whole Health, University Hospitals of Cleveland, Cleveland, Ohio, USA ${ }^{2}$ RCS, VA Puget Sound Health Care System, Seattle, Washington, USA

${ }^{3}$ Rehabilitation Care Services, VA Puget Sound Health Care

System American Lake Campus, Tacoma, Washington, USA

${ }^{4}$ Lakeside Hospital Library, University Hospitals of Cleveland, Cleveland, Ohio, USA

Correspondence to Dr Robert James Trager; Robert.Trager@UHhospitals.org

\section{ABSTRACT}

Introduction There are limited available research and guidance regarding the use of spinal manipulative therapy (SMT) in patients with low back-related symptoms following lumbar spine surgery, a condition called persistent spinal pain syndrome type 2 (PSPS-2). This publication outlines a review protocol to identify and synthesise individual participant data (IPD) to examine associations between patient, clinical and surgical variables and SMT application in adults with PSPS-2. Methods and analysis PubMed, OVID, Web of Science, Scopus, PEDro, Index to Chiropractic Literature and KoreaMed will be searched from inception to 1 January 2022 without language restrictions. Case reports, series, observational studies and cases from grey literature of adults receiving SMT for PSPS-2 will be included. Two investigators will independently screen citations, abstracts and full-text articles. A risk-of-bias assessment will be performed in duplicate to rate cases according to exposure and outcome ascertainment and data completeness. Data extraction will be performed in duplicate and missing IPD will be requested from corresponding authors. Multiple binary logistic regression will be used to identify independent predictors of the use of lumbar-SMT, lumbarmanual-thrust SMT and SMT within 1-year postsurgery. Patient, clinical and surgical variables will be summarised using descriptive statistics, while SMT-related outcomes (lumbar-SMT, lumbar-manual-thrust SMT and 1-year surgery-to-SMT interval) will be described using adjusted ORs with $95 \%$ Cls.

Ethics and dissemination This study was deemed not human subjects research by the University Hospitals' institutional review board. The results of this review will be disseminated at conferences and/or published in a peerreviewed journal.

PROSPERO registration number CRD42021250039.

\section{INTRODUCTION}

\section{Rationale}

Persistent spinal pain syndrome (PSPS) describes chronic or recurrent pain of spinal origin, including axial and/or radicular
Strengths and limitations of this study

- This will be the first study to investigate the influence of individual patient characteristics and clinical and surgical variables on the application of spinal manipulative therapy in patients with prior lumbar surgery.

- This review includes an analysis of individual patient data which will improve the ability to control for confounding variables using regression models.

- This review will use a comprehensive search strategy without language restrictions which will maximise the number of included cases.

- A limited amount of available research and unavailable individual patient data among included cases may hinder the ability of this review to identify significant findings.

symptoms. This syndrome is divided into PSPS type 1 , in which there is no prior relevant surgery, and persistent spinal pain syndrome type 2 (PSPS-2), in which patients have a history of spinal surgery. ${ }^{1}$ The latter category includes cases in which surgery directly or indirectly caused symptoms, was unsuccessful, was successful but symptoms recurred, and there is no relationship between surgery and symptoms, or this association is unclear. ${ }^{1}$ The term PSPS was recently proposed by an international group to replace 'failed back surgery syndrome' (FBSS) and to provide a label more inclusive of other terms such as postlaminectomy syndrome, adjacent-segment degeneration, chronic spinal pain after surgery, and postsurgical spine syndrome. ${ }^{1}$

\section{Description of the condition}

A systematic review found that PSPS-2 occurs in $4 \%-50 \%$ of patients following lumbar surgery and has various aetiologies, the most 
common being lumbar stenosis and disc herniation. ${ }^{2}$ Treatment for PSPS-2 usually begins with conservative methods such as strengthening, range of motion exercises and pharmacological therapy. ${ }^{3}$ Other options include neuromodulation, epidural injections, adhesiolysis and radiofrequency ablation. ${ }^{34}$ Revision surgery generally has a low success rate and is considered in cases refractory to treatment. ${ }^{4}$

Lumbar surgeries and anatomical variation can influence the number of lumbar motion segments (joints that allow motion), which is conceptually relevant to PSPS-2. While the lumbar spine typically has five motion segments (L1-2, L2-3, L3-4, L4-5 and L5-S1), ${ }^{5}$ this number varies in $5 \%-7 \%$ of individuals as a result of lumbosacral transitional vertebrae. ${ }^{6}$ Individuals with an additional mobile lumbar vertebra (L6) have six motion segments, while those with only four mobile lumbar vertebrae have four motion segments.

Surgical lumbar fusion eliminates motion segments, effectively increasing adjacent-segment loading and range of motion. ${ }^{7}$ Accordingly, a systematic review identified multilevel fixation as a risk factor for adjacent-segment disease. ${ }^{8}$ A reduced number of lumbar motion segments are also associated with sacroiliac joint pain. ${ }^{9}$ Conversely, evidence suggests motion-preserving surgeries such as disc arthroplasty are associated with a lower rate of adjacent-segment degeneration. ${ }^{8}$

The influence of spinal implants on spinal biomechanics may impact clinician decision-making to use spinal manipulative therapy (SMT). Each biomaterial has unique biomechanical properties which influences its tendency towards failure. For example, pedicle screws may loosen or break. ${ }^{10}$ While some implants promote bony fusion, others, such as artificial disc replacements, preserve segmental mobility. ${ }^{10}$ Imaging may identify postoperative instability or surgical instrumentation dysfunction and has been recommended as a precursor to SMT in patients with prior lumbar surgery. ${ }^{11} 12$

\section{Description of the intervention}

SMT can be defined broadly as manual therapy of the spinal vertebrae or joints, excluding soft tissue techniques such as massage. ${ }^{13}$ Although terminology can differ internationally and interprofessionally, SMT can be generally dichotomised into thrust or non-thrust techniques. ${ }^{1415}$ Thrust SMT involves a single high-velocity, low-amplitude (HVLA) impulse, while non-thrust SMT can involve multiple low-velocity motions/oscillations, such as low-grade joint mobilisation ${ }^{13} 1516$ or distraction techniques. ${ }^{1417}$ Thrust SMT can be further characterised according to manual (hands-on) or instrument-assisted delivery. $^{18}$

Although force magnitude of thrust SMT varies, ${ }^{19}$ and force alone does not distinguish thrust from nonthrust-SMT, $^{15}$ non-thrust-SMT is occasionally recommended as a gentler technique when there are SMT precautions such as hypermobility or osteoporosis. ${ }^{14} 17$ In addition, instrument-assisted-SMT (eg, Activator) is often perceived as a safe treatment option, potentially being used in cases where there are precautions to manualthrust SMT. 20

Research is limited regarding the application of SMT in PSPS-2. ${ }^{21-23}$ One recent systematic review identified multiple patients who benefited from SMT following discectomy, laminectomy, fusion, disc replacement and/ or spinal cord stimulator placement, but found inconclusive evidence to recommend for or against SMT in these patients. $^{22}$ Another review found insufficient evidence supporting the use of joint mobilisation postlumbar fusion. $^{21}$

Educational materials provide differing recommendations of SMT technique for patients postlumbar surgery, such as advising mobilisation away from the surgical site, ${ }^{24}$ treatment modification, ${ }^{25}$ avoidance of lower lumbar rotation $^{26}$ or no passive range of motion for 3 months at levels affected by surgery. ${ }^{27}$ Certain textbooks and continuing education bodies list manipulation under anaesthesia as a treatment for FBSS (PSPS-2), ${ }^{28}{ }^{29}$ provided patients do not have hypermobile segments and benefited from other forms of SMT. ${ }^{29}$ One orthopaedic textbook noted SMT 'seldom succeeds' in patients with prior laminectomy, although there was 'no harm' in trying $\mathrm{SMT}^{30}$

Precautions to SMT for patients with congenitally reduced lumbar motion segments due to block vertebrae $^{31-33}$ and lumbosacral transitional vertebrae ${ }^{34}$ may apply to patients with surgical lumbar fusion. For example, it has been argued that it is pointless to direct SMT towards congenitally fused vertebrae, which have no interposed motion segment. ${ }^{32}$ In addition, segments adjacent to congenital fusions may degenerate and become hypermobile, ${ }^{35}$ which can preclude SMT. $^{32}$ One survey of chiropractors in a large integrative healthcare organisation found less than half of respondents 'frequently' or 'sometimes' used lumbar HVLA-SMT in patients with prior lumbar fusion. ${ }^{36}$

It is less clear how laminectomies or discectomies influence SMT application, as these surgeries preserve segmental mobility. In one study, $76 \%$ of members of a small interdisciplinary panel rated SMT as 'appropriate' for patients with previous laminectomy. ${ }^{37}$ Ultimately, more research is needed to elucidate the standard of care and to develop clinical practice guidelines and prediction rules for SMT in PSPS-2.

Although patients with PSPS-2 may be managed conservatively, potentially using SMT, there is no clear consensus for the use and administration of SMT in these cases. Considering this knowledge gap, this systematic review of individual patient data aimed to identify potential associations between the characteristics of patients with PSPS-2 and SMT decision-making, and to provide guidance for clinicians incorporating SMT in their treatment of such patients.

\section{Objectives}

1. Identify independent predictors of use of lumbarSMT, lumbar-manual-thrust SMT, and timing of SMT 
in adults with PSPS-2 using multiple binary logistic regression models. We hypothesise that markers of reduced clinical and surgical complexity such as younger age, localised symptoms, increased number of motion segments, and absence of spinal implants will be independent predictors of greater odds of each of the three outcomes of interest: (1) lumbar-SMT, (2) lumbarmanual-thrust SMT and (3) <1-year surgery-to-SMT interval. We also hypothesise that chiropractors will have increased odds of using lumbar manual-thrust SMT relative to other disciplines.

2. Describe the characteristics of adults with PSPS-2 receiving SMT, including age, below-gluteal-fold symptoms, type of surgery, lumbar motion segments, spinal implants, postsurgical radiographs, SMT type, surgeryto-SMT interval and SMT practitioner.

\section{METHODS}

\section{Study design}

This review was designed in accordance with the Preferred Reporting Items for Systematic Review and Meta-Analysis Protocols $^{38}$ and related Preferred Reporting Items for Systematic Review and Meta-Analysis individual participant data (PRISMA-IPD) statements. ${ }^{39}$ Prior to registering this review, the Cochrane Database of Systematic Reviews and the International Prospective Register of Systematic Reviews (PROSPERO) were searched to ensure no similar meta-analyses were registered.

This review is registered with PROSPERO (CRD42021250039) with anticipated completion on 1 March 2022.

\section{Sample size calculation}

Sample size was calculated using a rule-of-thumb estimate for at least 10 events per variable for multiple logistic regression. ${ }^{40}$ Regression models in this study will include eight independent variables, excepting the single dependent variable in each model, yielding a required sample of 80 patients. This sample appeared feasible based on a prior review including patients receiving SMT following lumbar surgery. ${ }^{22}$

\section{Eligibility criteria}

Population

Included articles must describe patient(s) aged 18-89 with PSPS-2, with persistent spinal pain in the context of previous spinal surgery. ${ }^{1}$ An age cut-off of 89 was used as an individual's age 90 and above is considered a patient identifier by the Health Insurance Portability and Accountability Act, ${ }^{41}$ and external individual participant data (IPD) requests are planned. ${ }^{41}$ Included articles must describe axial or radicular low back disorders such as localised back pain, radiculopathy, sciatica or sacroiliac joint pain. Patients with cervical, thoracic or sacroiliac surgery without lumbar surgery will be excluded. Patients having only non-spinal surgeries (eg, hip and shoulder) will be excluded. Animal studies will be excluded. Patients with radiofrequency, thermal or cryoablation procedures and no other lumbar surgery (eg, intradiscal electrothermal therapy and basivertebral nerve ablation) will be excluded. Patients with spinal cord stimulator implantation will be included.

\section{Interventions}

Included articles must describe patients undergoing SMT for any part of the spine or pelvis. Articles describing intraoperative SMT will be excluded.

\section{Comparison}

This review will not require a comparator or control group for included studies as we expect there will be no control group available in nearly all included study types, and we are studying factors related to clinical decision-making for SMT use (ie, patient, clinical and surgical characteristics) as opposed to SMT efficacy or effectiveness.

\section{Outcome}

Articles describing patients with a positive or equivocal response to care will be included. Cases describing a serious adverse event such as a fracture, haematoma, cauda equina syndrome or severe worsening of symptoms ${ }^{42}$ will not be included. Cases with an adverse outcome could confound results considering a potential lack of clinical decision-making or dismissal of red flags that would normally preclude treatment. Although adverse-event cases could provide a real-world perspective, the goal of this study was to investigate if there are commonalities in the clinical decision-making among providers treating PSPS-2 in a prudent, safe and potentially beneficial manner.

\section{Study design}

Case reports and series and observational studies will be sought as these better reflect usual practice-based care and are more likely to provide IPD. Randomised controlled trials will not be included, considering these often exclude patients with prior surgery, ${ }^{43}$ treating providers would be less likely to use a pragmatic treatment approach with individualised clinical decision-making, and IPD such as the number of motion segments would likely be unavailable.

\section{Information sources}

This review will search the electronic databases: PubMed, OVID, Web of Science, Scopus, PEDro, the Index to Chiropractic Literature (ICL) and KoreaMed from inception until 1 January 2022 without language restrictions. Due to an expected paucity of literature, this review will also include individual cases from the grey literature including book chapters, conference abstracts and posters, provided they meet other selection criteria. Pertinent National Board of Chiropractic Examiners reference texts ${ }^{44}$ will be hand-searched. Grey literature will be chiefly sourced from the ICL, which catalogues conference material, as well as from hand-searching National Board of Chiropractic Examiners reference textbooks, ${ }^{44}$ and citation tracking. 


\begin{tabular}{|c|c|}
\hline Treatment strategy & $\begin{array}{l}\text { Prior procedure and } \\
\text { condition/region }\end{array}$ \\
\hline Tuina[tiab] OR & “Total disc replacement"[mesh] \\
\hline "Tui na"[tiab] OR & OR \\
\hline Chuna[tiab] OR & Disc replacement ${ }^{\star}[$ tiab] OR \\
\hline Flexion distraction[tiab] OR & Disk replacement*[tiab] OR \\
\hline Cox technique[tiab] OR & Disc arthroplast[tiab] OR \\
\hline Hvla[tiab] OR & Disk arthroplast*[tiab] OR \\
\hline High velocity low & Cementoplasty[mesh] OR \\
\hline amplitude[tiab] OR & cementoplast $^{*}[$ tiab] OR \\
\hline Instrument assisted & vertebroplast $^{\star}[$ tiab] OR \\
\hline adjust $^{\star}[$ tiab] OR & kyphoplast*[tiab] OR \\
\hline Instrument assisted & Diskectomy[mesh] OR \\
\hline manipulation*[tiab] OR & discectom[tiab] OR \\
\hline Joint manipulation* ${ }^{\star}[$ tiab] OR & diskectom*[tiab] OR \\
\hline Joint mobilisation*[tiab] OR & Extreme lateral interbody \\
\hline Joint mobilisation*[tiab] OR & fusion*[tiab] OR \\
\hline Manipulation under & Xlif[tiab] OR \\
\hline anaesthesia[tiab] OR & Foraminotomy[mesh] OR \\
\hline Fibrosis release[tiab] OR & foraminotom*[tiab] OR \\
\hline "Musculoskeletal & Interlaminar lumbar \\
\hline manipulations"[mesh] OR & instrumented fusion*[tiab] OR \\
\hline Musculoskeletal & Ilif[tiab] OR \\
\hline manipulation*[tiab] OR & Interlaminar implant* [tiab] OR \\
\hline Manipulative & Intrathecal drug delivery[tiab] \\
\hline rehabilitation*[tiab] OR & OR \\
\hline Manipulative therap*[tiab] & Itdd[tiab] OR \\
\hline OR & Laminectomy[mesh] OR \\
\hline Manual therap*[tiab] OR & laminectom*[tiab] OR \\
\hline Mobilization therap*[tiab] & laminotom*[tiab] OR \\
\hline OR & Laser surger ${ }^{\star}[$ tiab] OR \\
\hline Mobilisation therap*[tiab] & microdiskectom*[tiab] OR \\
\hline OR & microdiscectom*[tiab] OR \\
\hline Orthopedic & Persistent spinal pain \\
\hline manipulation*[tiab] OR & syndrome[tiab] OR \\
\hline Orthopaedic & Postoperative[tiab] OR \\
\hline manipulation*[tiab] OR & Post-operative[tiab] OR \\
\hline Osteopathic & Postsurgical[tiab] OR \\
\hline manipulation*[tiab] OR & Postsurgical[tiab] OR \\
\hline Chiropractic & "Spinal cord stimulation"[mesh] \\
\hline manipulation*[tiab] OR & OR \\
\hline Soft tissue & Spinal cord stimulat*[tiab] OR \\
\hline mobilisation*[tiab] OR & Adjacent segment disease[tiab] \\
\hline Soft tissue & OR \\
\hline mobilisation*[tiab] OR & "Failed back surgery \\
\hline Spinal manipulation*[tiab] & syndrome"[mesh] OR \\
\hline OR & Failed back surgery \\
\hline Spinal mobilisation*[tiab] & syndrome*[tiab] OR \\
\hline OR & Fbss[tiab] OR \\
\hline \multirow[t]{3}{*}{ Spinal mobilisation ${ }^{\star}[$ tiab] } & Junction failure*[tiab] OR \\
\hline & Recurrent disc*[tiab] OR \\
\hline & Recurrent disk*[tiab] \\
\hline
\end{tabular}

\section{Search strategy}

Three coauthors, including a research librarian (ACS), designed the search strategy. A list of terms (table 1) was adapted from a previous systematic review on the topic of manual therapies for patients with prior lumbar surgery. ${ }^{22}$ Changes included removal of terms related to soft-tissue therapies and stretching, and addition of terms relating

\section{Box 1 Reasons for exclusion at full-text screening}

\section{Exclusion criteria}

Patient $<18$ or $>89$ years old

No prior lumbar spine surgery

SMT not provided

No individual participant data available in study published before 1980

to traditional East Asian SMT (eg, chuna and tuina). The search was broadened by combining terms for low back conditions and prior procedures with a Boolean 'OR' as opposed to searching for these separately.

This review will not exclude articles based on language or severity of low back-related symptoms. Google Translate will be used to translate non-English abstracts and full-text articles, which has been found to be accurate for the purpose of systematic reviews and thought to help reduce English-language bias. ${ }^{45}$ In addition, SMT is used worldwide, ${ }^{13}$ and part of the study objectives was to determine if there were practitioner-related differences in the SMT approach to PSPS-2.

\section{Study records}

Data management

A two-stage study selection will be managed with Covidence systematic review software (Veritas Health Innovation, Australia). Data will be extracted into a predesigned Microsoft Excel Workbook. Multiple binary logistic regressions will be performed using GNU PSPP Statistical Analysis Software V.1.0.1. References will be managed in Endnote V.X9.

\section{Study selection process}

Two independent reviewers (RJT and CJD) will perform the initial screening of titles and abstracts of potentially eligible studies, and those meeting selection criteria will be saved. If there is insufficient information during initial screening to determine study eligibility, the full text will be obtained and reviewed. A screening checklist for titles/abstracts will be provided to reviewers (table 2 and box 1). Additional references will be sought by contacting experts in the field, asking coinvestigators to contribute articles not identified by the search strategy, screening the references of included articles and hand-searching textbooks.

\begin{tabular}{lll}
\hline Table 2 Screening checklist for titles/abstracts & \\
\hline Criteria* & Yes & No \\
\hline Patient $\geq 18$ and $\leq 89$ years old & $\square$ & $\square$ \\
Prior lumbar spine surgery & $\square$ & $\square$ \\
$\begin{array}{l}\text { Spinal manipulative therapy potentially } \\
\text { provided (any type) }\end{array}$ & $\square$ & $\square$
\end{tabular}

Case report, series or observational study $\quad \square \quad \square$

*All criteria must be 'yes' to pass first screening phase. 
Full texts will be obtained and reviewed independently by at least two reviewers against study selection criteria. Articles not meeting criteria will be excluded and the reason for exclusion will be recorded. Disagreements regarding initial and full-text screening will be resolved through discussion and/or involvement of a third reviewer.

\section{Data collection process}

To ensure feasibility of data extraction, the authorship team discussed articles from a prior related review ${ }^{22}$ that were likely eligible for the current review. ${ }^{46-48}$ Two investigators (RJT and KWM) will independently extract data from included studies. Disagreements will be resolved through discussion and/or involvement of a third investigator. If IPD is missing or requires clarification, coinvestigators will contact study corresponding authors to provide additional data, provided the study was published after 1980.

Requests for deidentified IPD will be made to study corresponding authors using an institutional email address, with sensitivity to the data sharing policy of the study organisations (online supplemental file 1). These authors will be offered an acknowledgement in the final review publication. Data requests will be limited to IPD needed for the study. Three attempts to obtain IPD will be made for authors not responding to inquiries, as multiple attempts are often required. ${ }^{49}$

\section{Data items}

Characteristics of included publications will be listed in tabular format. Graphic displays will summarise the frequency of surgery types, treating provider degrees and number of motion segments. The mean patient age, frequency of male patients, spinal implants, postsurgical imaging, lumbar-SMT, lumbar-manual-thrust SMT and $<1$-year surgery-to-SMT interval will be summarised. An IPD spreadsheet and risk-of-bias assessments will be published with study results, in-text or as a supplementary file. The synthesis of primary outcomes (ie, influence on lumbar-SMT, lumbar-manual-thrust SMT, surgeryto-SMT interval) will be provided as adjusted ORs with 95\% CIs as recommended for meta-analyses of dichotomous variables. ${ }^{50}$ In order for data to be accurately pooled, variables will be listed according to a common format and renamed for consistency (table 3 ).

Due to the paucity of literature on this subject, there was a need for a parsimonious regression model with a limited number of important variables, which would allow the primary study objectives to be sufficiently powered. Predictor variables were included in regression models based on their suspected importance in influencing SMT application. This was achieved by discussion between coinvestigators and solicitation of feedback from colleagues with experience in treating postsurgical patients (see the Acknowledgements section). Nine colleagues were solicited to rank potential variables in order of importance with respect to SMT application and were given the option to add variables and to provide feedback. In addition, only predictors expected to be reported consistently in included cases were chosen.

Ultimately, multiple predictors were omitted from the regression models: patient sex, presence of neurological deficits, number of prior surgeries, bone mineral density, previous response to SMT (eg, negative or positive), response to a premanipulative loading test, discomfort or inability of the patient to be positioned for SMT, receipt of acupuncture and/or dry needling, receipt of exercise therapies or other manual therapies, and receipt of nutraceuticals. The description of prior surgery was also omitted as variability between cases may preclude categorisation for regression analysis. Instead, other variables describe the surgery in a standardised method (ie, number of motion segments and presence of a spinal implant).

\section{Outcomes and prioritisation}

As a primary outcome, cases will be dichotomised according to lumbar-SMT, defined herein as SMT using a lumbar spine contact (eg, L1, L2, L3, L4 or L5) or creating movement at lumbar segments by contacting an adjacent structure (ie, the sacrum for lumbosacral junction manipulation or T12 causing motion between T12 and L1). 'Side-posture' or 'side-lying' SMT will be categorised as lumbar-SMT unless defined differently in the case, as these techniques result in a long-lever effect on the lumbar spine. ${ }^{51}$ Non-side-posture SMT performed by contacting the ilium to create motion at the sacroiliac joint will be categorised as non-lumbar-SMT. Patients receiving both lumbar and non-lumbar-SMT will be categorised as lumbar-SMT. The percentage of cases reporting lumbar-SMT, and an adjusted OR with 95\% CIs will be reported.

As another primary outcome, lumbar-SMT will be further characterised as manual-thrust SMT or nonmanual-thrust SMT (ie, not involving a manual thrust). 'Chiropractic manipulation' or 'chiropractic adjustments' will be categorised as manual-thrust SMT, which is traditionally used by this profession. ${ }^{51}$ Maitland grade $\mathrm{V}$ mobilisations will be considered manual-thrust SMT, while grades I-IV will be regarded as non-manual-thrust SMT. ${ }^{14}$ Manipulation under anaesthesia will be considered manual-thrust SMT. ${ }^{52}$ Side-posture or side-lying SMT will be categorised as lumbar manual-thrust SMT. Cases describing both manual-thrust and non-manualthrust SMT will be categorised as thrust SMT. Low-velocity manual-distraction techniques will be considered nonmanual-thrust SMT. ${ }^{53}$ Activator and other mechanical SMT instruments, which do not involve manual-thrust delivery, ${ }^{54}$ will be categorised as non-manual-thrust SMT. ${ }^{54}$ The percentage of cases reporting lumbar-manual-thrust SMT and an adjusted OR with 95\% CIs will be reported.

As another primary outcome, cases will be dichotomised according to a lumbar surgery-to-SMT interval of less than or greater than or equal to 1 year. We define this interval as the duration between the most recent lumbar 
Table 3 Variables extracted from studies

\begin{tabular}{|c|c|c|c|}
\hline Variables extracted & Potential values & Level of measurement & In regression models \\
\hline 1. First author surname & Text & NA & No \\
\hline 2. Year of publication & YYYY & NA & No \\
\hline 4. Patient sex & $\begin{array}{l}0=\text { male } \\
1=\text { female } \\
2=\text { unclear or other }\end{array}$ & Nominal & No \\
\hline $\begin{array}{l}\text { 6. A description of the surgical } \\
\text { intervention(s) }\end{array}$ & $\begin{array}{l}\text { Discectomy } \\
\text { Decompression (laminectomy/otomy, } \\
\text { facetectomy, foraminotomy) } \\
\text { Fusion } \\
\text { Arthroplasty (artificial disc) } \\
\text { Interspinous device } \\
\text { Spinal cord stimulator } \\
\text { Other (describe) } \\
\text {. Unclear }\end{array}$ & NA & No \\
\hline 7. Treating provider degree & $\begin{array}{l}0=\text { chiropractic } \\
1=\text { physical therapy } \\
2=\text { TEAM } \\
3=\text { MD/DO } \\
4=\text { other } \\
5=\text { unclear }\end{array}$ & Nominal & Yes \\
\hline $\begin{array}{l}\text { 8. Motion segments remaining } \\
\text { following surgery }\end{array}$ & $\begin{array}{l}0=0 \\
1=1 \\
2=2 \\
3=3 \\
4=4 \\
5=5 \text { or more } \\
6=\text { unclear }\end{array}$ & Ordinal & Yes \\
\hline 11. Lumbar-SMT & $\begin{array}{l}0=\text { no } \\
1=\text { yes } \\
2=\text { unclear }\end{array}$ & Nominal & Yes \\
\hline 12. Lumbar-manual-thrust SMT & $\begin{array}{l}0=\text { no } \\
1=\text { yes } \\
2=\text { unclear }\end{array}$ & Nominal & Yes \\
\hline 13. Surgery-to-SMT interval & $\begin{array}{l}0 \leq 1 \text { year } \\
1 \geq 1 \text { year } \\
2=\text { unclear }\end{array}$ & Nominal & Yes \\
\hline
\end{tabular}

MD/DO, doctor of medicine or osteopathy; SMT, spinal manipulative therapy; TEAM, traditional East Asian medicine.

surgery and SMT. One year was chosen based on this being a commonly studied time point for postlumbar surgery recovery, ${ }^{55} 56$ also an interval by which bony bridging should form postfusion ${ }^{57}$ (which could affect SMT selection) ${ }^{11}$ and to facilitate data extraction. The percentage of cases reporting a $<1$-year surgery-to-SMT interval and adjusted OR with 95\% CIs will be reported.

Cases will be dichotomised according to the presence of spinal implant(s), defined as the presence of any biomaterial introduced into the lumbar region (eg, cage, screws, rods, plates, disc replacement, spinal cord stimulator or other as listed in a prior publication. ${ }^{10}$ Filler used in cementoplasty and autograft and allograft bone will be considered spinal implants. Patients who previously had spinal implants which were removed preceding SMT will be considered as not having spinal implants.

Cases will be dichotomised according to presence of postsurgical imaging including radiographs, CT, MRI and nuclear medicine imaging studies. In addition, imaging must be subsequent to lumbar surgery and reviewed (visually or by examining a radiology report) by the SMT 
provider. Cases publishing images or details of postsurgical imaging will be considered as having imaging present.

Cases will be dichotomised according to below-glutealfold symptoms. This boundary has been used as a proxy for sciatica, disc herniation, radicular pain, radiculopathy and nerve root compression, ${ }^{52}$ and is used in this study as a marker of symptom severity, considering low backrelated lower extremity pain is associated with nerve root compression and lateral stenosis. ${ }^{58}$ We used the proximal gluteal-fold boundary instead of a more distal boundary because precise pain distributions may be unavailable. We also expect to identify cases of L3 or L4 radicular pain (eg, in patients with adjacent-segment degeneration), in which symptoms radiate into the thigh but not distal to the knee.

Provider degrees will be grouped according to scope of practice and similarity in practice approaches. Doctor of chiropractic degrees, including international bachelor's and masters' variations, will be combined. Likewise, physical therapy degrees will be grouped (eg, master or doctor of physical therapy). Osteopathic and medical doctor degrees will form another group. Traditional East Asian medical degrees will comprise another category (eg, oriental medicine, traditional Chinese medicine and traditional Korean medicine degrees ${ }^{59}$ Providers who do not fit into these categories will be labelled 'other'.

The number of mobile lumbar segments will be categorised as $0,1,2,3,4$ or ' 5 or more', to account for lumbosacral transitional vertebrae, ${ }^{34}$ with an additional category of 'unclear'. This categorisation was adapted from a prior study. ${ }^{9}$ This determination will be made during data extraction, based on the surgery description, imaging and imaging report if available. Palpation findings will not be used to calculate motion segments. For this variable, any capacity for segmental motion will suffice to be considered 'mobile'.

\section{Risk-of-bias in individual studies}

Two reviewers (RJT and CJD) will independently perform a risk-of-bias assessment. This will be applied to individual cases rather than at the study level as we expect patientlevel data to vary, with certain cases missing IPD or having limited exposure or outcome variables. Discrepancies will be resolved through mutual discussion and involvement of a third reviewer, if necessary.

Although a risk-of-bias assessment is recommended by the PRISMA-IPD statement, ${ }^{39}$ there is limited guidance on the quality assessment of case reports for this purpose. This study will use a flexible rubric applicable to case reports and series, and allows for omission of questions that do not apply to the study topic (Table 4).$^{60}$

The rubric in this study omits criteria designed for adverse drug events (eg, alternative causes, challengerechallenge and dose-response). We also omit the question regarding sufficient follow-up as this review is not analysing long-term outcomes of care but rather clinical interventions. Case selection was not used as a criterion as this review is expected to mostly include case reports and series, which have inherent publication bias. Use of this modified rubric will enable the exclusion of cases with unclear information during sensitivity analysis.

\section{Data synthesis}

IPD will be synthesised qualitatively and quantitatively in a one-stage manner. Use of IPD will facilitate the process of controlling for confounding variables during regression analyses. ${ }^{61}$ As we expect to synthesise mostly case reports and series, each with a limited number of patients, a twostage model of initially aggregating within-study data is unnecessary. Characteristics of each included study will be listed in tabular format. The proportion and/or mean with SD for each study variable will be calculated.

Bivariate correlation testing will be performed before logistic regression using a two-tailed Pearson correlation matrix to identify highly-associated variables, which will be discarded from regression models if the correlation coefficient is 0.7 or higher. The number of motion segments will be tested for a normal distribution by creating a histogram and using the Kolmogorov-Smirnov test; however, a non-normal distribution will not prohibit use of this variable in regressions. This study will include three multiple binary logistic regression models:

1. The dependent variable is lumbar-SMT (performed vs not performed), with covariates including belowgluteal-fold symptoms, provider degree, patient age, number of motion segments, spinal implant,

\begin{tabular}{ll}
\hline Table $4 \quad$ Risk-of-bias assessment \\
\hline Domain $\quad$ Leading explanatory questions \\
\hline Ascertainment & 1. Was the exposure adequately ascertained (eg, is it clear that symptoms were consistent with \\
& $\begin{array}{l}\text { PSPS-2)? } \\
\text { 2. Was the outcome adequately ascertained (eg, is it clear if manual-thrust SMT versus non-manual- } \\
\text { thrust SMT was provided)? }\end{array}$ \\
Reporting & $\begin{array}{l}\text { 3. Is the case described with sufficient details to allow other investigators to replicate the research } \\
\text { or to allow practitioners to make inferences related to their own practice? Study must have } 9 \text { of } 11\end{array}$ \\
& study variables present (not counting author, year and study title).
\end{tabular}

$3=$ high quality, $2=$ moderate quality, $\leq 1=$ low quality.

PSPS-2, persistent spinal pain syndrome type 2; SMT, spinal manipulative therapy. 
postsurgical imaging, lumbar-manual-thrust SMT and surgery-to-SMT interval.

2. The dependent variable is lumbar-manual-thrust SMT (performed vs not performed), with covariates including below-gluteal-fold symptoms, provider degree, patient age, number of motion segments, spinal implant, postsurgical imaging, lumbar-SMT and surgery-toSMT interval.

3 . The dependent variable is the surgery-to-SMT interval ( $<1$ or $\geq 1$ year), with covariates including below-glutealfold symptoms, provider degree, patient age, number of motion segments, spinal implant, postsurgical imaging, lumbar-SMT and lumbar manual-thrust SMT.

Data from cases without any IPD after author requests will be excluded from analysis. Studies meeting eligibility criteria with partial IPD will be included in initial analysis. Missing data will be designated a specific value and treated as a separate category in regression analysis. This will allow all possible cases to be included in the qualitative analysis and initial regression models, as few cases with complete IPD are expected.

A statistical method of confounder selection, such as backward-stepwise selection, will not be used because clinically important covariates were selected a priori based on content knowledge, and all of these predictors will be factored into regression models regardless. For the same reason, a univariate model will not be used. Our planned regression models will identify independent predictors of SMT use while weighing their relationship to other variables. This strategy should provide results applicable to real-world practice, as clinicians weigh the importance of multiple factors when choosing a treatment strategy. A fixed or random-effects model will not apply for our analysis because our methods involve a one-stage meta-analytical approach without a weighted average or summary statistic for individual studies.

\section{Meta-biases}

Reporting bias of included studies will be minimised by including unpublished IPD. This study will not use Egger's test or a funnel plot to detect publication bias because we expect the majority of included studies to be case reports, which have inherent publication bias. ${ }^{62}$

Although defining a separate category for missing values for regression analysis is a valid method, ${ }^{63}$ it could create biased results. We will examine the potential bias of this strategy via sensitivity analysis, in which regression models are repeated with cases missing excessive IPD ( $\geq 25 \%)$ or a high risk-of-bias ('low quality') excluded.

This review will not incorporate a forest plot as a summary of overall treatment effect is not planned. For this reason, we will not calculate an $\mathrm{I}^{2}$ for study heterogeneity. Likewise, exploration of variation in SMT effects is not planned.

\section{Confidence in cumulative evidence}

As this meta-analysis is expected to be based mostly on case reports and series, the certainty of evidence will remain low. ${ }^{60}$

\section{Ethics and dissemination}

This study was deemed not human subjects research by the University Hospitals' institutional review board. The results of this review will be disseminated at conferences and/or published in a peer reviewed journal.

Acknowledgements We wish to acknowledge the clinicians who provided valuable feedback that helped us select the covariates used in the regression models: Patrick Battaglia, DC; Joshua R S Browning, DC; Zachary A Cupler, DC, MS; Erica M Gaitley, DC, MS; Jordan A Gliedt, DC; Ross Mattox, DC; Sarah E Prosak DC; Christopher B Roecker, DC, MS; David A Vincent, DC.

Contributors RJT, CJD and JAD conceived, designed and coordinated the review protocol. ACS was responsible for designing the search strategy and screening methodology, RJT and KWM planned the data extraction methodology, and all authors contributed to writing the review protocol. RJT is the guarantor of this review.

Funding The authors have not declared a specific grant for this research from any funding agency in the public, commercial or not-for-profit sectors.

Competing interests RJT reports he has received book royalties from authoring two texts on the topic of sciatica.

Patient consent for publication Not applicable.

Ethics approval This study does not involve human participants.

Provenance and peer review Not commissioned; externally peer reviewed.

Supplemental material This content has been supplied by the author(s). It has not been vetted by BMJ Publishing Group Limited (BMJ) and may not have been peer-reviewed. Any opinions or recommendations discussed are solely those of the author(s) and are not endorsed by BMJ. BMJ disclaims all liability and responsibility arising from any reliance placed on the content. Where the content includes any translated material, BMJ does not warrant the accuracy and reliability of the translations (including but not limited to local regulations, clinical guidelines, terminology, drug names and drug dosages), and is not responsible for any error and/or omissions arising from translation and adaptation or otherwise.

Open access This is an open access article distributed in accordance with the Creative Commons Attribution Non Commercial (CC BY-NC 4.0) license, which permits others to distribute, remix, adapt, build upon this work non-commercially, and license their derivative works on different terms, provided the original work is properly cited, appropriate credit is given, any changes made indicated, and the use is non-commercial. See: http://creativecommons.org/licenses/by-nc/4.0/.

\section{ORCID iDs}

Robert James Trager http://orcid.org/0000-0002-4714-1076

Clinton J Daniels http://orcid.org/0000-0002-2727-6184

Kevin W Meyer http://orcid.org/0000-0002-9370-1644

Jeffery A Dusek http://orcid.org/0000-0001-9581-0564

\section{REFERENCES}

1 Christelis N, Simpson B, Russo M, et al. Persistent spinal pain syndrome: a proposal for failed back surgery syndrome and ICD-11. Pain Med 2021;22:807-18.

2 Clancy C, Quinn A, Wilson F. The aetiologies of failed back surgery syndrome: a systematic review. J Back Musculoskelet Rehabil 2017;30:395-402.

3 Daniell JR, Osti OL. Failed back surgery syndrome: a review article. Asian Spine J 2018;12:372-9.

4 Baber Z, Erdek M. Failed back surgery syndrome: current perspectives. J Pain Res 2016;9:979-87.

5 Louis R. Functional anatomy of the lumbar spine. In: The artificial disc. Springer, 1991: 3-11.

6 Apazidis A, Ricart PA, Diefenbach CM, et al. The prevalence of transitional vertebrae in the lumbar spine. Spine J 2011:11:858-62.

7 Nagata H, Schendel MJ, Transfeldt EE, et al. The effects of immobilization of long segments of the spine on the adjacent and distal facet force and lumbosacral motion. Spine 1993;18:2471-9.

8 Hashimoto K, Aizawa T, Kanno H, et al. Adjacent segment degeneration after fusion spinal surgery-a systematic review. Int Orthop 2019;43:987-93.

9 Unoki E, Abe E, Murai $\mathrm{H}$, et al. Fusion of multiple segments can increase the incidence of Sacroiliac joint pain after lumbar or lumbosacral fusion. Spine 2016;41:999-1005. 
10 Warburton A, Girdler SJ, Mikhail CM, et al. Biomaterials in spinal implants: a review. Neurospine 2020;17:101-10.

11 Daniels CJ, Wakefield PJ, Bub GA, et al. A narrative review of lumbar fusion surgery with relevance to chiropractic practice. J Chiropr Med 2016;15:259-71.

12 Taylor J. The Role of Radiography in Evaluating Subluxation. In: Mosby E, ed. Foundations of chiropractic: subluxation. 2nd edn, 2005: 115-32.

13 Hurwitz EL. Epidemiology: spinal manipulation utilization. Journal of Electromyography and Kinesiology 2012;22:648-54.

14 Scaringe J, Kawaoka C. Mobilization techniques. In: Principles and practice of chiropractic. 3rd edn. McGraw-Hill, 2005: 767-85.

15 Dvorák J, Dvorák V, Gilliar W. Definitions and principles of manual medicine diagnosis and treatment. In: Musculoskeletal manual medicine: diagnosis and treatment. 1st edn. Thieme, 2008: 4-5.

16 Hengeveld E, Banks K. Maitland's Vertebral Manipulation: Management of Neuromusculoskeletal Disorders. In: Elsevier health sciences. vol 1, 2013.

17 Bergmann TF, Peterson DH. Nonthrust procedures: mobilization traction, and soft tissue techniques. In: Chiropractic Technique. Elsevier Health Sciences 2010:381-418.

18 Hooper PD. Evolution and basic principles of the chiropractic adjustment and manipulation. In: Principles and practice of chiropractic. McGraw-Hill, 2005: 745-53.

19 Downie AS, Vemulpad S, Bull PW. Quantifying the high-velocity, low-amplitude spinal manipulative thrust: a systematic review. J Manipulative Physiol Ther 2010;33:542-53.

20 Read DT, Wilson FJH, Gemmell HA. Activator as a therapeutic instrument: survey of usage and opinions amongst members of the British chiropractic association. Clinical Chiropractic 2006;9:70-5.

21 Madera M, Brady J, Deily S, et al. The role of physical therapy and rehabilitation after lumbar fusion surgery for degenerative disease: a systematic review. J Neurosurg 2017;26:694-704.

22 Daniels CJ, Cupler ZA, Gliedt JA, et al. Manipulative and manual therapies in the management of patients with prior lumbar surgery: a systematic review. Complement Ther Clin Pract 2021;42:101261.

23 Tekmyster G, Jonely H, Lee DW, et al. Physical therapy considerations and recommendations for patients following spinal cord stimulator implant surgery. Neuromodulation 2021. doi:10.1111/ ner.13391. [Epub ahead of print: 05 Apr 2021]

24 Labbe A, Page P, Morris CE. Rehabilitation of the surgical lumbar spine patients. In: Low back syndromes: integrated clinical management. 1st edn. McGraw-Hill Education, 2006: 727-39.

25 Triano J. The theoretical basis for spinal manipulation. In: Principles and practice of chiropractic. 3rd edn. McGraw-Hill, 2005: 361-81.

26 Cox J. Biomechanics, Adjustment Procedures, Ancillary Therapies, and Clinical Outcomes of Cox Distraction Technique. In: Low back pain: mechanism, diagnosis and treatment. 7th edn. Lippincott Williams \& Wilkins, 2012.

27 Dakwar E, Deukmedjian A, Ritter Y. Spinal pathology, conditions, and deformities: Surgical intervention. In: Pathology and intervention in musculoskeletal rehabilitation. 2 edn, 2015.

28 Kohlbeck F. Medication-assisted spinal manipulation. In: Principles and practice of chiropractic. 3rd edn. McGraw-Hill, 2005: 841-59.

29 Gordon RC, ed. Manipulation Under Anesthesia. 1st ed. CRC Press, 2005.

30 Cyriax P, Cyriax JH. Cyriax's Illustrated Manual of Orthopaedic Medicine. 2nd edn. Butterworth-Heinemann, 1993.

31 Howe J. Imaging the Low Back. In: The low back and pelvis: clinical applications. Aspen Publishers, Inc, 1997.

32 Peterson CK, Gatterman MI. The nonmanipulable subluxation. In: Found Chiropr subluxation. 2nd edn. St Louis Mosby Yearb Inc, 2005: 168-90.

33 Souza T. The Geriatric Patient. In: Differential diagnosis and management for the Chiropractor. 5th ed. Jones \& Bartlett Learning, 2018: 1125-53.

34 Muir JM. Chiropractic management of a patient with low back pain and Castellvi type II lumbosacral transitional vertebrae. J Chiropr Med 2012;11:254-9.

35 Farshad-Amacker NA, Herzog RJ, Hughes AP, et al. Associations between lumbosacral transitional anatomy types and degeneration at the transitional and adjacent segments. Spine J 2015;15:1210-6.

36 Daniels CJ, Gliedt JA, Suri P, et al. Management of patients with prior lumbar fusion: a cross-sectional survey of Veterans Affairs chiropractors' attitudes, beliefs, and practices. Chiropr Man Therap 2020;28:1-10.

37 Herman PM, Hurwitz EL, Shekelle PG, et al. Clinical scenarios for which spinal mobilization and manipulation are considered by an expert panel to be inappropriate (and appropriate) for patients with chronic low back pain. Med Care 2019;57:391-8.
38 Moher D, Shamseer L, Clarke M, et al. Preferred reporting items for systematic review and meta-analysis protocols (PRISMA-P) 2015 statement. Syst Rev 2015;4:1.

39 Stewart LA, Clarke M, Rovers M, et al. Preferred reporting items for systematic review and meta-analyses of individual participant data: the PRISMA-IPD statement. JAMA 2015;313:1657-65.

40 Peduzzi P, Concato J, Kemper E, et al. A simulation study of the number of events per variable in logistic regression analysis. $J$ Clin Epidemiol 1996:49:1373-9.

41 HHS.gov. Guidance regarding methods for de-identification of protected health information in accordance with the health insurance portability and accountability act (HIPAA) privacy rule, 2012. Available: https://www.hhs.gov/hipaa/for-professionals/ privacy/special-topics/de-identification/index.html [Accessed 15 Oct 2021].

42 Hebert JJ, Stomski NJ, French SD, et al. Serious adverse events and spinal manipulative therapy of the low back region: a systematic review of cases. J Manipulative Physiol Ther 2015;38:677-91.

43 Amundsen PA, Evans DW, Rajendran D, et al. Inclusion and exclusion criteria used in non-specific low back pain trials: a review of randomised controlled trials published between 2006 and 2012. BMC Musculoskelet Disord 2018;19.

44 National board of chiropractic examiners (NBCE). Part III reference Texts. Available: https://mynbce.org/part-iii-reference-texts/ [Accessed 26 Feb 2021].

45 Jackson JL, Kuriyama A, Anton A, et al. The accuracy of Google translate for Abstracting data from Non-English-Language trials for systematic reviews. Ann Intern Med 2019;171:677-9.

46 Coulis CM, Lisi AJ. Chiropractic management of postoperative spine pain: a report of 3 cases. J Chiropr Med 2013;12:168-75.

47 Perrucci RM, Coulis CM. Chiropractic management of post spinal cord stimulator spine pain: a case report. Chiropr Man Therap 2017;25:1-7

48 O'Shaughnessy J, Drolet M, Roy JF. Chiropractic management of patients post-disc arthroplasty: eight case reports. Chiropr Osteopat 2010;18:1-7.

49 Ventresca M, Schünemann HJ, Macbeth F, et al. Obtaining and managing data sets for individual participant data meta-analysis: Scoping review and practical guide. BMC Med Res Methodol 2020;20:1-18.

50 Stewart GB, Altman DG, Askie LM, et al. Statistical analysis of individual participant data meta-analyses: a comparison of methods and recommendations for practice. PLoS One 2012;7:e46042.

51 Bergmann TF. High-velocity low-amplitude manipulative techniques. In: Principles and practice of chiropractic. 3rd edn. McGraw-Hill, 2005: 755-66.

52 Lin C-WC, Verwoerd AJH, Maher CG, et al. How is radiating leg pain defined in randomized controlled trials of conservative treatments in primary care? a systematic review. Eur J Pain 2014;18:455-64.

53 Gudavalli MR, Cox JM. Real-Time force feedback during flexiondistraction procedure for low back pain: a pilot study. J Can Chiropr Assoc 2014;58:193-200.

54 Fuhr AW. Low-force and instrument technique. In: Principles and practice of chiropractic. 3rd edn. McGraw-Hill, 2005: 787-803.

55 Staartjes VE, de Wispelaere MP, Vandertop WP, et al. Deep learning-based preoperative predictive analytics for patient-reported outcomes following lumbar discectomy: feasibility of center-specific modeling. Spine J 2019;19:853-61.

56 Khor S, Lavallee D, Cizik AM, et al. Development and validation of a prediction model for pain and functional outcomes after lumbar spine surgery. JAMA Surg 2018;153:634.

57 Young PM, Berquist TH, Bancroft LW, et al. Complications of spinal instrumentation. Radiographics 2007;27:775-89.

58 Beattie PF, Meyers SP, Stratford P, et al. Associations between patient report of symptoms and anatomic impairment visible on lumbar magnetic resonance imaging. Spine 2000;25:819-28.

59 Park H-L, Lee H-S, Shin B-C, et al. Traditional medicine in China, Korea, and Japan: a brief introduction and comparison. Evid Based Complement Alternat Med 2012;2012:1-9.

60 Murad MH, Sultan S, Haffar S, et al. Methodological quality and synthesis of case series and case reports. BMJ Evid Based Med 2018;23:60-3.

61 Thomas D, Radji S, Benedetti A. Systematic review of methods for individual patient data meta- analysis with binary outcomes. BMC Med Res Methodol 2014;14:1-9.

62 Nissen T, Wynn R. The clinical case report: a review of its merits and limitations. BMC Res Notes 2014;7:1-7.

63 Vittinghoff E, Glidden DV, Shiboski SC. Simple approaches to handling missing data. Regression Methods in Biostatistics: Linear, Logistic, Survival, and Repeated Measures Models 2012;442. 